\title{
Pattern electroretinograms become abnormal when background diabetic retinopathy deteriorates to a preproliferative stage: possible use as a screening test
}

\author{
G B ARDEN, ${ }^{12}$ A M P HAMILTON, ${ }^{2} \mathrm{~J}$ WILSON-HOLT, ${ }^{3}$ S RYAN, ${ }^{2}$ J S YUDKIN, \\ AND A KURTZ
}

From the 'Institute of Ophthalmology, and ${ }^{2}$ Moorfields Eye Hospital, City Road, London EC1; ${ }^{3}$ Western Ophthalmic Hospital, Marylebone Road, London NW1; ' Whittington Hospital, Archway, London N19; and the ${ }^{5}$ Middlesex Hospital, London WC1

SUMmaRY The pattern electroretinogram (PERG) and other tests were given to a selected group of patients with diabetes, ranging from those who had no retinopathy or funduscopic changes to those in the preproliferative state. None had visual symptoms. The PERG was found to be normal in patients with microaneurysms and a few blot haemorrhages. However, when cotton-wool spots and angiographic evidence of areas of capillary non-perfusion were present, the PERG was reduced below the normal value. Similar changes occurred with the oscillatory potentials of the ERG evoked by an intense flash, but the results were more variable. So in the individual patient the test is not a reliable indicator of the progress of the retinopathy. The value of the PERG in screening is discussed.

While the electroretinogram (ERG) evoked by diffuse flashes of light remains normal in cases of diabetic retinopathy unless gross retinal damage or vitreous haemorrhage has supervened, ${ }^{12}$ minor components of the electroretinogram, the oscillatory potentials (OPs) have been reported to be affected earlier ${ }^{34}$ and to be an indicator of the progression of the condition. ${ }^{5}$ The small pattern electroretinogram (PERG) evoked by reversing a black and white pattern may be produced in a different manner from the normal ERG ${ }^{6-x}$ and has been found to be reduced by retinal diseases which do not affect the ERG itself. ${ }^{-14}$ In a preliminary' study of the PERG in the eyes of patients who were about to have argon laser panretinal photocoagulation for advanced diabetic retinopathy (Arden, unpublished) it was found that the PERG was uniformly abnormal. We have therefore recorded PERGs from the eyes of diabetics with varying degrees of milder retinopathy, and now report that, while mild background retinopathy does not affect the PERG in every case of a small series, the response is abnormal in the presence of fundu-

Correspondence to Professor G B Arden, Institute of Ophthalmology, Judd Street, London WC1H 9QS. scopic and angiographic evidence of capillary nonperfusion despite the absence of visual symptoms.

\section{Patients and methods}

Patients were drawn from the diabetic clinics of the Middlesex Hospital and the Whittington Hospital, either by consultation of computer records or by searching the notes. The criteria for entry into the trial were (a) no cardiovascular, neurological, or other ocular disease should be known to be present, and there should be no eye symptoms; $(b)$ the corrected visual acuity should be better than $6 / 12$; (c) the age of onset of diabetes should be no greater than 50 ; and $(d)$ no result would be included if the eye had diabetic retinopathy which required immediate laser treatment.

The reason for these requirements was to eliminate patients with unrelated conditions which themselves might affect the PERG. The most common of these are uncorrected refractive error, glaucoma, amblyopia, cataract, optic neuritis, degenerative maculopathies, and hypertensive eye disease-and proliferative diabetic retinopathy itself. As a result of the selection almost all the patients had diabetes of 
juvenile onset and required insulin injections, though a number of them developed the condition in early adult life. We attempted to select patients with all grades of retinopathy, including those with normal fundi.

The patients selected were interviewed by the staff of the diabetic clinics, and the nature of the proposed investigation and what was involved for each patient was explained. Informed consent obtained, the patient was then referred to the Electrodiagnostic Clinic, Moorfields Eye Hospital, where electrophysiological investigations were performed. The PERG was usually recorded from one eye, without pupillary dilatation, under the standard conditions described in the caption to Fig. 1 and in previous papers. When there was an interocular difference, the eye with the better acuity was tested. Oscillatory potentials were obtained on the fellow eye after pupillary dilatation as described by Bresnick et al., and the records were measured in the way those authors specify. In one case the fellow eye had proliferative retinopathy, so the result was not included. In one patient the PERG was obtained without the patient's wearing spectacles to correct myopia, and this result was discarded. In some cases both tests were done on each eye and the two results averaged. The recording system consisted of a specially built amplifier, under the control of a PDP11/23 computer programmed to average and display the results. The system was driven by interrupts from the TV pattern generator. Ophthalmological investigations were performed and the degree of disease classified without reference to the electrodiagnostic findings, and, since a number of patients were thought to have no retinopathy, the investigation was essentially 'double blind'. Colour photographs of the fundus were obtained on all patients, and the region photographed (in those patients with fundus abnormalities) were specified after funduscopy. Only in those patients with ophthalmologically normal fundi or those in whom only a few microaneurysms were visible was fluorescein angiography omitted.

On the basis of the photographs the patients were divided into four groups: I normal fundus; II few microaneurysms only; III microaneurysms, a few
Fig. 1 Pattern ERGs (PERGs) and oscillatory potentials (OPs) from the eyes of a subject with grade III retinopathy (see Fig. 2) and (right) grade IV disease (see Fig. 2). $P E R G$ s are the computer calculated average of three records, each derived from 150-200 repetitions of the stimulus. Artefact rejection was incorporated. The response was recorded with a gold foil electrode on the cornea, an indifferent electrode of the ipsilateral temple near the lateral canthus, and a forehead earth electrode. The stimulus consisted of $30^{\prime}$ squares displayed on a TV monitor which subtended $15 \times 22^{\circ}$ at $1 \mathrm{~m}$, and had a mean luminance of $100 \mathrm{~cd} / \mathrm{m}^{2}$. The pattern reversed eight times per second. The contrast was nearly $100 \%$. The white surround to the monitor was illuminated to $200 \mathrm{~cd} / \mathrm{m}^{2}$. The OPs were obtained by light from a $40 \mathrm{~J}$ photographic flash tube, cycle time 20 s, delivered to the eye through a rigid Perspex light pipe $2.5 \mathrm{~cm}$ diameter, the end of which was placed less than $1 \mathrm{~cm}$ from the cornea. Electrodes were as for the PERG. The eye was preadapted for two or more flashes, and then the responses to the succeeding four flashes were averaged. This sequence was repeated three times. The dashed lines drawn show the method of measurement: two troughs were joined and the peak height estimated at the times indicated by arrows from the baseline thus formed. The voltages shown in Table 1 are the sum of the first five oscillations (following Bresnick et al. ${ }^{5}$ ) for the OPs. A similar procedure was followed for the PERGs, but there was only one peak to measure. The responses are chosen to be average amplitude for groups III and IV. 


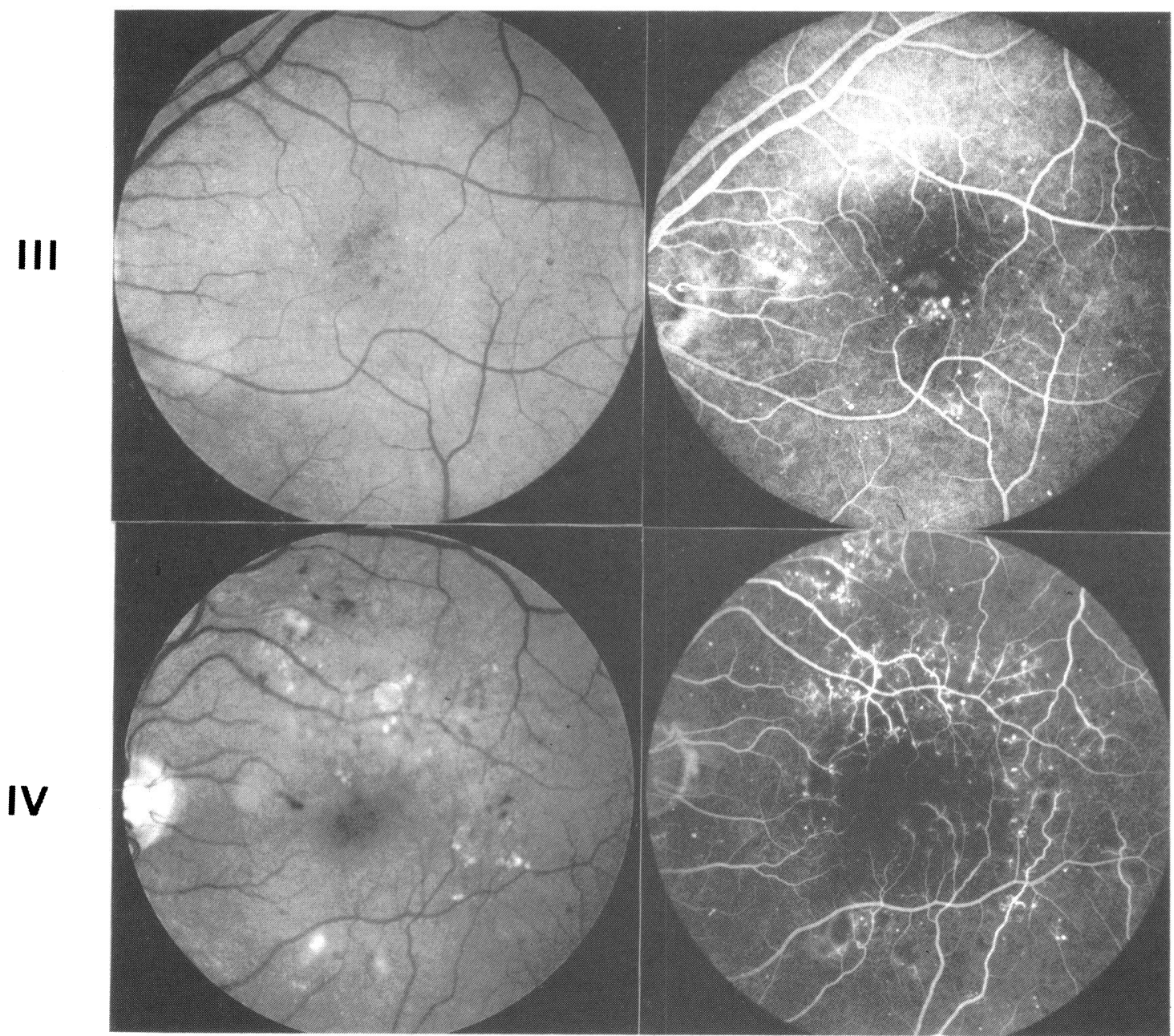

Fig. 2 Fundus photographs and angiograms of a representative patient from groups III and IV. The patient from group III had a PERG of $4 \cdot 2 \mu V$. The patient whose fundus illustrates group IV produced the largest PERGs of the group $-2 \cdot 3 \mu V$. Note that not only are there more microaneurysms and cotton-wool spots in his fundus, but also there are darker areas on the angiogram which indicate regions where capillary non-perfusion is occurring: such areas are absent in the angiograms of group III.

small 'blot' haemorrhages, and/or hard exudates, but not further changes; no evidence of capillary nonperfusion on angiography; IV the above, plus cottonwool spots, pronounced leakage, and evidence of capillary non-perfusion on angiography.

In the absence of angiography it is possible that patients classified as group I might include some from group II. No patient in group IV had proliferative retinopathy. There were no new vessels at the disc or peripherally. The aim in classification was to separate patients who had background retinopathy of a level not requiring surgical intervention (group III) from those in whom surgical intervention might be required in the near future. Characteristic changes are shown in Fig. 2.

\section{Results}

Table 1 summarises the results. The mean PERG value for group I was larger than was previously found for a group of normal persons ${ }^{13}$ but not significantly so, and this was probably associated with the fact that the patients in this group were younger and had very good eyesight. The OPs were in the range reported by previous workers. ${ }^{5}$ The remaining groups were similar in their ages and duration of 
Table 1 Comparison of findings in four subgroups of diabetics

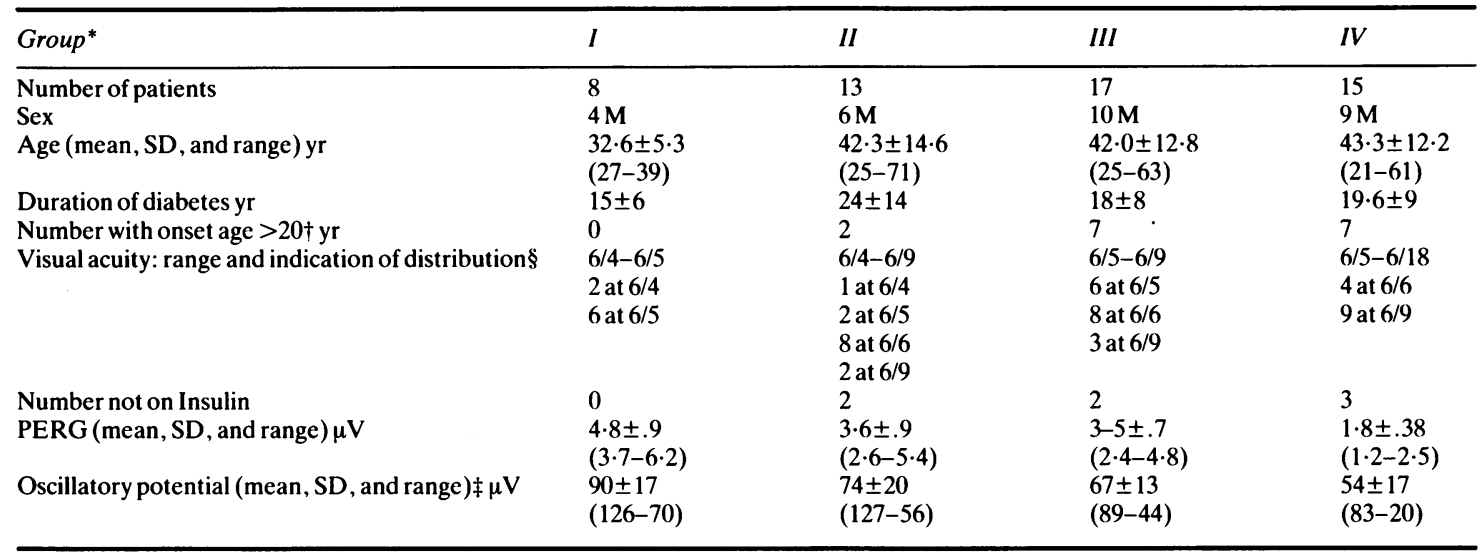

${ }^{*}$ See text for definition.

†Data not available for one patient in groups I and II, three in group III, two in group IV.

$\ddagger$ Data not available for one in group III, three in group IV.

§If not shown, extreme values for visual acuity obtained in one patient only.

disease. In groups II and III the mean PERG value was nearly exactly that found previously for normal subjects. There was a slight progressive decrease in the OPs, so that the mean OP in group III was less than the lower limit of normal given by Bresnick $e t$ al. ${ }^{5}$ Group IV, with more advanced disease, did not differ from the groups II and III in terms of age or duration or type of disease, but the visual acuity was on the whole slightly reduced. In only one case was the reduction great enough to be counted as outside the normal limit. In this patient perimacular retinal changes were evident, but there was no obvious macular oedema. There was a very striking and highly significant reduction in the mean PERG voltage. The probability of the patients in groups III and IV coming from the same population was less than 0.02 by Students $t$ test. The mean value in group IV was half that in group III, and significantly below the value ( 2 standard deviations below the mean) accepted in the clinic as the lower bound of normal $(2.4 \mu \mathrm{V}$ for persons under 50 years of age). In one case in this group, a patient aged 30 , the value was the average of that for both eyes, individually 2.2 and $2 \cdot 7 \mu \mathrm{V}$. In two more cases the value was $2.3 \mu \mathrm{V}$ (ages 38 and 49). In all other cases the value was $<2 \mu \mathrm{V}$.

The oscillatory potentials appeared to behave in a slightly different manner. There was a reduction in the mean values for each successive group, which was not so exaggerated for group IV. The scatter of results was larger, and therefore the mean result, and the result in any individual, was not significant. The mean age of patients in group IV was rather larger than for the other groups, but the duration of diabetes was not greater.

\section{Discussion}

While sensitive tests of retinal function such as contrast sensitivity may show abnormalities due to diabetic retinopathy, ${ }^{15}$ they tend to have a high incidence of false positive results, unless the criteria for abnormality are set so that there is an unacceptable level of false negative results, or the test is made so detailed and prolonged that it is clinically inapplicable. For this reason the report that OPs are abnormal in the deteriorating diabetic retina was of considerable interest because the test is quick to administer.

The results on the OPs are the first we have obtained with this technique. We have no normal population with which we can compare our results. The patients without retinopathy have OPs of the size previously reported. ${ }^{5}$. If we use the lower limit of normal determined elsewhere, then none of group I are abnormal, 7 of group II, 10 of group III, and 11 of group IV are abnormal. There is no significant distinction between the group averages or the proportions of individuals abnormal between groups. The average OPs deteriorate, as previously reported, but do so in the presence of mild background retinopathy. Thus the result is not very useful in the individual.

The present results suggest that the PERG provides more reliable data than the OPs. However, we have recorded PERGs in our clinic for some years, and the test is done very frequently. Apart from the small scatter in the results the reduction in the PERG appears to come about at the particular phase of the disease when referral to an ophthalmologist becomes necessary. 
In several retinal conditions the PERG is grossly reduced even when the disease is mild..$^{1314}$ In cases of inherited juvenile maculopathy, disease of a severity which causes any reduction in visual acuity has a gross effect on the PERG. However, the relationship cannot be simple, because refractive errors which cause reduction in visual acuity produce a much smaller effect on the PERG. The patients in this series did not suffer specifically from diabetic maculopathy, and loss of visual acuity even in group IV was minimal. However, in this group there are very severe reductions in the PERG, which strongly suggests that the abnormality in the PERG appears when the capillary circulation is affected, and not earlier. In view of the fact that the PERG tests the function of the central retina, the gross reduction occurring in all cases in group IV at first seems surprising, since the areas of non-perfusion may occur in the peripheral retina. However in a separate study $^{16}$ of preproliferative retinopathy with peripheral non-perfusion it has been found that Amsler chart testing shows transient metamorphism, and fluorescein angiography shows evidence of microvascular abnormalities in the posterior pole. It would seem therefore that there are often minor changes at the posterior pole in patients whose main pathology consists of peripheral capillary closure, and this may be causing the change in the PERG.

Although the series is small, there is little overlap between groups III and IV. Even on the basis of one test all the patients of group IV could be distinguished from those of III, if we allow the worse eye of the patient for whom we have results in both eyes. Even if the patients are judged on the severest criteria, which make no allowance for age variation, only three of group IV could be considered normal, and no one in the other groups would be abnormal. If the PERG was introduced for screening diabetics, serial tests would be done on each patient, and it should thus be possible to spot deterioration in any individual, even if the decrease was not so gross as appears in Table 1. Some of the individuals in group IV may have benefitted from immediate surgical intervention, but in no case was it mandatory, and therefore it is likely that, in some cases at least, serial testing might show a repeated PERG abnormality before the patient was in danger of losing vision. Advanced warning of deterioration would therefore be possible, as shown in the follow up findings with the OPs.'

The method of patient selection may have biased the results, so it is not wise to speculate about the applicability of the test to patients in general. There are two important differences between the patients tested and the general population. Firstly, we have excluded anything except the mildest form of diabetic maculopathy by the entry criteria, which demanded a normal visual acuity. However, in other maculopathies the PERG has been found to decrease early in the disease, ${ }^{13}$ and there is no reason to suppose that this will not be the case in diabetes. Secondly, although in this series we attempted to exclude anyone with any pre-existing eye condition, diabetes is found mainly in older people, in whom eye disease is prevalent. The progressive development of a cataract should cause a reduction in the PERG unrelated to a retinopathy, because the effective contrast in the stimulus is reduced by a cataract (Hamilton AM, Urey $\mathrm{H}$, in preparation). If the patient did not have an adequate spectacle correction, this too would affect the PERG. Thus interpretation of test results in the unselected individual patient may require additional clinical examination.

However, the PERG has certain advantages as a screening test. The patient's pupils do not need to be dilated, so there are no sequelae to the test, as there are with OP testing or funduscopy, which require mydriasis. In co-operative patients the actual recording time need be no greater than a minute or two; introducing the patient to the test, and attaching the electrodes takes longer than the test itself. Specialised equipment is required, but it is a fraction of the cost of that needed for fundus photography.

\section{References}

1 Gjotterberg $M$. The electroretinogram in diabetic retionopathy: a cinical study and critical survey. Acta Ophthalmol (Kbh) 1974; 52: 521-33.

2 Perleman I, Gdal-on M, Miller B, Zonis S. Neural function of the diabetic retina after argon laser photocoagulation assessed electroretinographically. Br J Ophthalmol 1985; 69: 240-6.

3 Simonsen SE. Electroretinographic study of diabetics: preliminary report. Acta Ophthalmol (Kbh) 1965; 43: 841-3.

4 Simonsen SE. Prognostic value of ERG (oscillatory potential) in juvenile diabetics. Acta Ophthalmol (Kbh) 1975; 123 (suppl): 223-4.

5 Bresnick GH, Korth K, Groo A, Palta M. Electroretinographic oscillatory potentials predict progression of diabetic retinopathy. Arch Ophthalmol 1984; 102: 1307-11.

6 Arden GB, Vaegan. Differences between the focal and pattern electroretinogram in man. J Physiol (Lond) 1982; 327: 67-68P.

7 Arden GB, Vaegan. Electroretinograms evoked in man by local uniform or patterned stimulation. J Physiol (Lond) 1983; 341: 85-104.

8 Maffei L, Fiorentini A. Electroretinographic responses to alternating gratings before and after section of the optic nerve. Science 1981; 211: 953-5.

9 Arden GB, Vaegan, Hogg CR, Powell D, Carter RM. Pattern ERGs are abnormal in many amblyopes. Trans Ophthalmol Soc UK 1980; 100: 452-60.

10 Fiorentini A, Maffei L, Pirchio M, Spinelli D, Porciatti V. The ERG response to alternating gratings in patients with diseases of the peripheral retinal pathway. Invest Ophthalmol Vis Sci 1981; 21: $490-3$.

11 Bobak P, Bodis-Wollner I, Harnois C, et al. Pattern electroretinograms and visual evoked potentials in glaucoma and multiple sclerosis. Am J Ophthalmol 1983; 96: 72-83.

12 Wanger P, Person HS. Pattern reversal electroretinograms in 
unilateral glaucoma. Invest Ophthalmol Vis Sci 1983; 24: 749-53.

13 Arden GB, Carter RM, Macfarlan A. Pattern and Ganzfeld electroretinograms in macular disease. Br J Ophthalmol 1984; 68: $878-84$.

14 Vacgan, Arden GB, Hogg CR. Properties of normal electroretionograms cvoked by pattern stimuli in man. Abnormalities in optic nerve disease and amblyopia. Doc Ophthalmol Proc Ser 1982; 31: 111-29.

15 Arden GB. Doyne Memorial Lecture. Trans Ophthalmol Soc UK 1978; 98: 219-31.

Accepted for publication 10 October 1985. 